\title{
CONTROLLING MANIFOLD COVERS OF ORBIFOLDS
}

\author{
D. B. McReYNOLDS
}

\begin{abstract}
In this article we prove a generalization of Selberg's lemma on the existence of torsion free, finite index subgroups of arithmetic groups. Some of the geometric applications are the resolution a conjecture of Nimershiem and answers to questions of Long-Reid and the author.
\end{abstract}

\section{Introduction}

For a compact orbifold $M$ with an infinite fundamental group, there is no reason to expect $M$ to possess a finite manifold cover. Indeed, even the existence of a finite orbifold cover cannot be guaranteed. However, when $\pi_{1}^{\text {orb }}(M)$ admits a faithful linear representation, Selberg's lemma (see for instance [2]) furnishes $M$ with many finite manifold covers. Given their prolificacy, one might ask more geometrically of these covers. Explicitly, we ask the following pair of questions.

Question 1. If $N$ is a properly immersed, $\pi_{1}$-injective submanifold of $M$, can $N$ be lifted to an embedded submanifold in a finite manifold cover of $M$ ?

Question 2. If $N$ is an immersed, totally geodesic submanifold of $M$, can $N$ to be lifted to an embedded submanifold in a finite manifold cover of $M$ ?

This article aims at resolving the first question in some special cases. In the final section, we partially address the second question. Even in these special situations, there are some new geometric applications. We have elected to postpone the motivation for these geometric results until Section 6. Before describing them, we give an abbreviated account of the associated algebraic problem.

The enterprize of promoting immersions to embeddings in finite covers has received some attention in recent years. The associated algebraic problem for subgroups $\pi_{1}(N)$ of $\pi_{1}^{\text {orb }}(M)$ is directly related to subgroup separability (see 20]). In this vein, we proved in [15] a result that promotes $\pi_{1}$-injective immersions to embeddings in finite covers when $M$ is arithmetic and $N$ is infranil. Our present goal is to ensure the cover constructed in [15] can be taken to be a manifold. Algebraically, this requires a torsion free, finite index subgroup $\Lambda_{0}$ of $\pi_{1}^{o r b}(M)$ that contains $\pi_{1}(N)$. The main result of this article is the resolution of this problem. Throughout the remainder of this article, $[\eta]$ will denote the $\operatorname{GL}(n, \mathbf{Z})$-conjugacy class of an element $\eta$ of $\operatorname{GL}(n, \mathbf{Z})$.

Theorem 1.1. Let $\eta \in \mathrm{GL}(n, \mathbf{Z})$ be a semisimple element and $\Gamma<\mathrm{GL}(n, \mathbf{Z})$ a torsion free, virtually unipotent subgroup. Then there exists a finite index subgroup $\Lambda_{0}$ of $\mathrm{GL}(n, \mathbf{Z})$ such that $\Gamma<\Lambda_{0}$ and $[\eta] \cap \Lambda_{0}=\emptyset$.

Received by the editors August 12, 2008.

The author was partially supported by an NSF postdoctoral fellowship. 
Corollary 1.2. Let $\Gamma<\mathrm{GL}(n, \mathbf{Z})$ be a torsion free, virtually unipotent subgroup. Then there exists a torsion free, finite index subgroup $\Lambda_{0}$ of $\mathrm{GL}(n, \mathbf{Z})$ such that $\Gamma<\Lambda_{0}$.

The main geometric application of Corollary 1.2 given here is on the structure of cusp cross sections of arithmetic orbifolds and manifolds. Specifically, using the aforementioned subgroup separability result [15, Theorem 3.1] in tandem with Corollary 5.3 (see Section 5), we can promote $\pi_{1}$-injective immersions of infranil manifolds into arithmetic orbifolds to embeddings in some finite manifold cover of the target orbifold. With this and our previous work in [15, 16, we can derive a few new geometric results. The first verifies a conjecture of Nimershiem [18, Conjecture 2'].

Theorem 1.3 (Nimershiem's conjecture). Let $M$ be a closed flat $n$-manifold. Then the space of similarity classes of flat structures on $M$ that can be realized in cusp cross sections of (arithmetic) hyperbolic $(n+1)$-manifolds is dense in the space of flat similarity classes.

This was previously known only for $n=1,2$, and 3 (see [18). The following corollary of Theorem 1.3 was also previously known only for small $n$.

Corollary 1.4. Every closed flat n-manifold is diffeomorphic to a cusp cross section of an arithmetic hyperbolic $(n+1)$-manifold.

Corollary 1.4 upgrades the main result of Long-Reid [12, Theorem 1.1] to manifolds, answering a question implicitly asked by Long and Reid [12, p. 286] (Nimershiem [18, Conjecture 1'] also conjectured this without an arithmetic assumption).

Our next result is the extension of Theorem 1.3 to the complex and quaternionic hyperbolic settings via [16. Theorem 3.5] and Corollary 5.3

Theorem 1.5. $\quad$ (a) Let $N$ be a closed almost flat manifold modeled on the Heisenberg group $\mathfrak{N}_{2 n+1}$. Then the space of similarity classes of almost flat metrics on $N$ that can be realized in cusp cross sections of complex hyperbolic $(n+1)-$ manifolds is either empty or dense in the space of almost flat metrics.

(b) Let $N$ be a closed almost flat manifold modeled on the quaternionic Heisenberg group $\mathfrak{N}_{4 n+3}$. Then the space of similarity classes of almost flat metrics on $N$ that can be realized in cusp cross sections of quaternionic hyperbolic $(n+1)-$ manifolds is either empty or dense in the space of almost flat metrics.

In [15, Theorem 5.4], we gave a necessary and sufficient condition on when this set is non-empty. This provides the following corollary which answers a question asked in [15, Section 8].

Corollary 1.6. Every closed Nil 3-manifold is diffeomorphic to a cusp cross section of an arithmetic complex hyperbolic 2-manifold.

Density for almost flat structures on compact Nil 3-manifolds follows from Theorem 1.5 (a) and Corollary 1.6. Our final result is the geometric consequence of Corollary 5.3 .

Corollary 1.7. Let $N$ be a closed infranil manifold and $X$ an arithmetic orbifold. Then any proper $\pi_{1}$-injective immersion of $N$ into $X$ can be lifted to be an embedding in a finite manifold cover of $X$. 
Note that Theorem 1.1 (see Theorem 5.2 permits one to find finite manifold covers of $X$ such that any finite number of closed geodesics fail to lift and $N$ can be lifted to be embedded.

\section{Preliminaries}

Notation. For each prime $p, \mathbf{Z}_{p}, \mathbf{Q}_{p}$ will denote the $p$-adic integers and field, respectively. The full profinite closure of $\mathbf{Z}$ will be denoted by $\widehat{\mathbf{Z}}$. Associated to these topological rings are the topological groups $\operatorname{GL}\left(n, \mathbf{Z}_{p}\right), \operatorname{GL}\left(n, \mathbf{Q}_{p}\right)$, and $\operatorname{GL}(n, \widehat{\mathbf{Z}})$. Finally, the reduction homomorphism given by reducing coefficients modulo $m$ will be denoted by $r_{m}: \operatorname{GL}(n, \mathbf{Z}) \rightarrow \mathrm{GL}(n, \mathbf{Z} / m \mathbf{Z})$.

2.1. Given a subgroup $\Gamma$ of $\operatorname{GL}(n, \mathbf{Z})$, we denote the closure of $\Gamma$ in $\operatorname{GL}\left(n, \mathbf{Z}_{p}\right)$ by $\mathrm{Cl}_{p}(\Gamma)$ and its closure in $\mathrm{GL}(n, \widehat{\mathbf{Z}})$ by $\mathrm{Cl}(\Gamma)$. The following is a restatement of [15, Theorem 3.1].

Theorem 2.1. If $\Gamma<\mathrm{GL}(n, \mathbf{Z})$ is virtually solvable, then $\mathrm{Cl}(\Gamma) \cap \mathrm{GL}(n, \mathbf{Z})=\Gamma$.

2.2. Given an element $\gamma$ in $\operatorname{GL}(n, \mathbf{Z})$, there exists a unique decomposition $\gamma=\gamma_{s} \gamma_{u}$ called the Jordan decomposition. The elements $\gamma_{s}, \gamma_{u} \in \operatorname{GL}(n, \mathbf{C})$ have the following properties:

(1) $\gamma_{s}$ is diagonalizable and $\gamma_{u}-I_{n}$ is nilpotent.

(2) $\left[\gamma_{s}, \gamma_{u}\right]=\gamma_{s}^{-1} \gamma_{u}^{-1} \gamma_{s} \gamma_{u}=I_{n}$.

An element $\gamma$ is called semisimple if $\gamma_{u}=I_{n}$ and unipotent if $\gamma_{s}=I_{n}$. It will be our convention to consider the trivial element as unipotent. Whether or not an element is semisimple or unipotent is a conjugacy invariant, a fact gleamed from the formulae

$$
\left(\eta^{-1} \gamma \eta\right)_{s}=\eta^{-1} \gamma_{s} \eta, \quad\left(\eta^{-1} \gamma \eta\right)_{u}=\eta^{-1} \gamma_{u} \eta
$$

2.3. A subgroup $\Gamma$ of $\operatorname{GL}(n, \mathbf{C})$ is unipotent if $\Gamma$ is conjugate in $\operatorname{GL}(n, \mathbf{C})$ into the group of upper triangular matrices with ones along the diagonal. More generally, if $\Gamma$ has a finite index subgroup that is unipotent, we say that $\Gamma$ is virtually unipotent.

Given a virtually unipotent subgroup $\Gamma$ of $\operatorname{GL}(n, \mathbf{Z})$, each element $\gamma$ in $\Gamma$ possesses a Jordan decomposition $\gamma_{s} \gamma_{u}$. As some power of $\gamma$ is unipotent, $\gamma^{m}=\gamma_{u}^{m}$ where $m$ is the order of $\gamma_{s}$. In the event that $\Gamma$ is torsion free, $\gamma_{u}$ is necessarily nontrivial and hence no element of $\Gamma$ can be semisimple. Note also that both $\gamma_{s}, \gamma_{u}$ reside in $\mathrm{GL}(n, \mathbf{Q})$.

2.4. Associated to $\Gamma$ is the set of semisimple factors

$$
\operatorname{Semi}(\Gamma)=\left\{\gamma_{s}: \gamma \in \Gamma\right\} \subset \operatorname{GL}(n ; \mathbf{C}) .
$$

According to (1), the conjugate action of $\Gamma$ induces an action on the set $\operatorname{Semi}(\Gamma)$. The finiteness of the quotient $\operatorname{Semi}(\Gamma) / \Gamma$ under this action will be critical.

Lemma 2.2. If $\Gamma<\mathrm{GL}(n, \mathbf{Z})$ is virtually unipotent, then $\operatorname{Semi}(\Gamma) / \Gamma$ is finite.

We postpone the proof of Lemma 2.2 until Section 4. For future reference, we fix a complete set of representatives ${ }^{s} \gamma_{1}, \ldots,{ }^{s} \gamma_{r} \in \operatorname{Semi}(\Gamma)$ for the quotient set $\operatorname{Semi}(\Gamma) / \Gamma$. 


\section{Proof of Theorem 1.1}

In this section we prove Theorem 1.1 We begin by deducing Corollary 1.2 from Theorem 1.1.

Proof of Corollary 1.2. A well known corollary of Weil local rigidity is the finiteness of conjugacy classes of torsion elements in $\operatorname{GL}(n, \mathbf{Z})$ (see for instance [19, VII.5]). Let $\eta_{1}, \ldots, \eta_{t}$ be a complete set of representatives for these conjugacy classes of torsion elements. According to Theorem 1.1, for each $\eta_{j}$, there exists a finite index subgroup $\Lambda_{j}$ of $\operatorname{GL}(n, \mathbf{Z})$ such that $\Gamma<\Lambda_{j}$ and $\left[\eta_{j}\right] \cap \Lambda_{j}=\emptyset$. The subgroup

$$
\Lambda_{0}=\bigcap_{j=1}^{t} \Lambda_{j},
$$

is easily seen to suffice for verifying the corollary.

3.1. Proof of Theorem 1.1. In this subsection, we prove Theorem 1.1. The proof is elementary (modulo Lemma 2.2), relying only Jordan form and passage to convergent subsequence (via compactness).

3.1.1. Some basic facts. We begin by recording some elementary facts, the proofs of which have been included for completeness.

Lemma 3.1. If $\gamma$ is a limit of unipotent elements in $\mathrm{GL}\left(n, \mathbf{Z}_{p}\right)$, then $\gamma$ is unipotent.

Proof. Let $\left\{\gamma_{j}\right\}$ be a sequence of unipotent elements in $\operatorname{GL}\left(n, \mathbf{Z}_{p}\right)$ that converge to $\gamma$. As there exists a uniform bound on the multiplicative order of $\gamma_{j}-I_{n}$, it follows that $\gamma$ is unipotent. Specifically, if $N$ is an integer such that the multiplicative order of $\gamma_{j}-I_{n}$ is bounded above by $N$ for all $j$, it follows that for all $j>0,\left(\gamma_{j}-I_{n}\right)^{N}=0_{n}$. Thus

$$
0_{n}=\lim _{j}\left(\left(\gamma_{j}-I_{n}\right)^{N}\right)=\left(\lim _{j}\left(\gamma_{j}-I_{n}\right)\right)^{N}=\left(\left(\lim _{j} \gamma_{j}\right)-I_{n}\right)^{N}=\left(\gamma-I_{n}\right)^{N} .
$$

Lemma 3.2. If $\eta \in \mathrm{GL}(n, \mathbf{Z})$ is semisimple, then $\mathrm{Cl}_{p}([\eta])$ consists of semisimple elements.

Proof. For $\lambda \in \mathrm{Cl}_{p}([\eta])$, there exists a convergent sequence $\left\{\eta_{j}^{\prime}\right\}$ in $[\eta]$ whose limit is $\lambda$. For each $\eta_{j}^{\prime}$, by definition there exists $\beta_{j} \in \mathrm{GL}(n, \mathbf{Z})$ such that $\beta_{j}^{-1} \eta_{j}^{\prime} \beta_{j}=\eta$. As $\left\{\beta_{j}\right\}$ is a sequence in the compact group $\operatorname{GL}\left(n, \mathbf{Z}_{p}\right)$, there exists a convergent subsequence $\left\{\beta_{\ell}\right\}$ of $\left\{\beta_{j}\right\}$ with limit $\beta \in \mathrm{GL}\left(n, \mathbf{Z}_{p}\right)$. Note that by continuity of taking inverses, the sequence $\left\{\beta_{\ell}^{-1}\right\}$ is also convergent and has limit $\beta^{-1}$. In total, this yields

$$
\eta=\lim _{\ell} \eta=\lim _{\ell}\left(\beta_{\ell}^{-1} \eta_{\ell}^{\prime} \beta_{\ell}\right)=\left(\lim _{\ell} \beta_{\ell}^{-1}\right) \cdot\left(\lim _{\ell} \eta_{\ell}^{\prime}\right) \cdot\left(\lim _{\ell} \beta_{\ell}\right)=\beta^{-1} \lambda \beta .
$$

As $\eta$ is semisimple, $\lambda$ is as well.

Lemma 3.3. For subsets $R_{1}, R_{2} \subset \mathrm{GL}(n, \mathbf{Z})$, if $\mathrm{Cl}_{p}\left(R_{1}\right) \cap \mathrm{Cl}_{p}\left(R_{2}\right)=\emptyset$, then there exists a positive integer $K$ such that $r_{p^{K}}\left(R_{1}\right) \cap r_{p^{K}}\left(R_{2}\right)=\emptyset$. 
Proof. Note that as the closed sets $\mathrm{Cl}_{p}\left(R_{1}\right)$ and $\mathrm{Cl}_{p}\left(R_{2}\right)$ are disjoint, the topological normality of $\operatorname{GL}\left(n, \mathbf{Z}_{p}\right)$ implies that we can find open subsets $O_{j}$ of $\operatorname{GL}\left(n, \mathbf{Z}_{p}\right)$ that contain $\mathrm{Cl}_{p}\left(R_{j}\right)$ and are disjoint from $\mathrm{Cl}_{p}\left(R_{k}\right)$ where $j \neq k$. The subsets $\mathrm{Cl}_{p}\left(r_{p^{\ell}}^{-1}\left(r_{p^{\ell}}\left(R_{j}\right)\right)\right)$ are open (and closed) in $\operatorname{GL}\left(n, \mathbf{Z}_{p}\right)$, contain $\mathrm{Cl}_{p}\left(R_{j}\right)$, and have the feature that

$$
\bigcap_{\ell=1}^{\infty} \mathrm{Cl}_{p}\left(r_{p^{\ell}}^{-1}\left(r_{p^{\ell}}\left(R_{j}\right)\right)\right)=\mathrm{Cl}_{p}\left(R_{j}\right) .
$$

Therefore, for some large integer $K$, it must be that

$$
\mathrm{Cl}_{p}\left(r_{p^{K}}^{-1}\left(r_{p^{K}}\left(R_{1}\right)\right)\right) \cap \mathrm{Cl}_{p}\left(r_{p^{K}}^{-1}\left(r_{p^{K}}\left(R_{2}\right)\right)\right)=\emptyset .
$$

Thus, we must have the less restrictive, desired conclusion

$$
r_{p^{K}}\left(R_{1}\right) \cap r_{p^{K}}\left(R_{2}\right)=\emptyset .
$$

3.1.2. Limit point criterion. For the statement of the following proposition, recall by Lemma 2.2 that there exists a finite set $\left\{{ }^{s} \gamma_{1}, \ldots,{ }^{s} \gamma_{r}\right\}$ of semisimple factors up to $\Gamma$-conjugation.

Proposition 3.4. If $\eta \in \mathrm{Cl}_{p}(\Gamma)$ is semisimple, then there exists $1 \leq k_{\eta} \leq r$ such that ${ }^{s} \gamma_{k_{\eta}} \in \mathrm{Cl}_{p}(\Gamma)$.

Proof. By definition, there exists a convergent sequence $\left\{\gamma_{j}\right\}$ in $\Gamma$ with limit $\eta$. Consider the pair of sequences ${ }^{s} \gamma_{j}=\left(\gamma_{j}\right)_{s},{ }^{u} \gamma_{j}=\left(\gamma_{j}\right)_{u}$. We will first prove the proposition under the assumption that ${ }^{s} \gamma_{j}={ }^{s} \gamma_{k_{\eta}}$ for all $j$ and some fixed $k_{\eta}$. We will see below that the general situation can be reduced to this. Under the assumption that ${ }^{s} \gamma_{j}={ }^{s} \gamma_{k_{\eta}}$, the associated unipotent factor sequence $\left\{{ }^{u} \gamma_{j}\right\}$ of $\left\{\gamma_{j}\right\}$ must also converge since ${ }^{u} \gamma_{j}=\gamma_{j}\left({ }^{s} \gamma_{k_{\eta}}\right)^{-1}$. Suggestively setting $\eta_{u}$ to be the limit of the sequence $\left\{{ }^{u} \gamma_{j}\right\}$, we assert that ${ }^{s} \gamma_{k_{\eta}} \eta_{u}$ is the Jordan decomposition for $\eta$. That $\eta_{u}$ is unipotent follows from Lemma 3.1 (we already know that ${ }^{s} \gamma_{k_{\eta}}$ is semisimple). To see that ${ }^{s} \gamma_{k_{\eta}} \eta_{u}=\eta$, notice that ${ }^{s} \gamma_{k_{\eta}} \cdot{ }^{u} \gamma_{j}=\gamma_{j}$. Therefore,

$$
\eta=\lim _{j} \gamma_{j}=\lim _{j}\left({ }^{s} \gamma_{k_{\eta}} \cdot{ }^{u} \gamma_{j}\right)={ }^{s} \gamma_{k_{\eta}} \cdot\left(\lim _{j}{ }^{u} \gamma_{j}\right)={ }^{s} \gamma_{k_{\eta}} \cdot \eta_{u} .
$$

Finally to see that $\left[{ }^{s} \gamma_{k_{\eta}}, \eta_{u}\right]=I_{n}$, note that

$$
\begin{aligned}
I_{n} & =\lim _{j}\left[{ }^{s} \gamma_{k_{\eta}},{ }^{u} \gamma_{j}\right]=\lim _{j}\left(\left({ }^{s} \gamma_{k_{\eta}}\right)-1 \cdot\left({ }^{u} \gamma_{j}\right)-1 \cdot{ }^{s} \gamma_{k_{\eta}} \cdot{ }^{u} \gamma_{j}\right) \\
& =\left({ }^{s} \gamma_{k_{\eta}}\right)^{-1} \cdot\left(\lim _{j}\left({ }^{u} \gamma_{j}\right)^{-1}\right) \cdot{ }^{s} \gamma_{k_{\eta}} \cdot\left(\lim _{j}{ }^{u} \gamma_{j}\right) \\
& =\left({ }^{s} \gamma_{k_{\eta}}\right)^{-1} \cdot \eta_{u}^{-1} \cdot{ }^{s} \gamma_{k_{\eta}} \cdot \eta_{u}=\left[{ }^{s} \gamma_{k_{\eta}}, \eta_{u}\right]
\end{aligned}
$$

as needed. This shows that ${ }^{s} \gamma_{k_{\eta}} \eta_{u}$ is the Jordan decomposition for $\eta$. As $\eta$ is semisimple, it must be that $\eta_{u}=I_{n}$ and hence $\eta={ }^{s} \gamma_{k_{\eta}}$ for some $k_{\eta}$.

It could be the case that the semisimple factor sequence ${ }^{s} \gamma_{j}$ for $\gamma_{j}$ is not constant. Using Lemma 2.2, we will reduce this case to the previous one. To begin, by Lemma 2.2 , there exists a sequence $\left\{\alpha_{j}\right\}$ in $\Gamma$ such that

$$
\left(\alpha_{j}^{-1} \gamma_{j} \alpha_{j}\right)_{s}=\alpha_{j}^{-1}{ }^{s} \gamma_{j} \alpha_{j}={ }^{s} \gamma_{k_{j}}, \quad k_{j} \in\{1, \ldots, r\} .
$$


In particular, some $k_{\eta}$ must occur infinitely often and so we can pass to a subsequence $\gamma_{i}$ such that

$$
\left(\alpha_{i}^{-1} \gamma_{i} \alpha_{i}\right)_{s}={ }^{s} \gamma_{k_{\eta}}
$$

for some fixed $1 \leq k_{\eta} \leq r$. As $\left\{\alpha_{i}\right\}$ is a sequence in the compact group $\mathrm{Cl}_{p}(\Gamma),\left\{\alpha_{i}\right\}$ has a convergent subsequence $\left\{\alpha_{\ell}\right\}$ with limit $\alpha \in \mathrm{Cl}_{p}(\Gamma)$. Again by continuity of taking inverses, $\left\{\alpha_{\ell}^{-1}\right\}$ is convergent with limit $\alpha^{-1} \in \mathrm{Cl}_{p}(\Gamma)$. In total, we see now that

$$
\lim _{\ell} \alpha_{\ell}^{-1} \gamma_{\ell} \alpha_{\ell}=\left(\lim _{\ell} \alpha_{\ell}^{-1}\right) \cdot\left(\lim _{\ell} \gamma_{\ell}\right) \cdot\left(\lim _{\ell} \alpha_{\ell}\right)=\alpha^{-1} \eta \alpha .
$$

As $\alpha, \alpha^{-1}, \eta \in \mathrm{Cl}_{p}(\Gamma)$, so is $\alpha^{-1} \eta \alpha$. In addition, since $\eta$ is semisimple, so is its conjugate $\alpha^{-1} \eta \alpha$. By taking $\alpha^{-1} \eta \alpha$ instead of $\eta$, we can assume that $\eta$ is the limit of a sequence $\left\{\gamma_{j}\right\}$ in $\Gamma$ whose semisimple factors are constant.

Remark. By construction, $\eta$ and ${ }^{s} \gamma_{k_{\eta}}$ are conjugate in $\operatorname{GL}\left(n, \mathbf{Z}_{p}\right)$.

3.1.3. Avoiding a semisimple factor. As before, the elements ${ }^{s} \gamma_{1}, \ldots,{ }^{s} \gamma_{r}$ are a complete list of semisimple factors up to $\Gamma$-conjugation given by Lemma 2.2 .

Lemma 3.5. For each $k=1, \ldots, r$, there exists a prime $p_{k}$ such that ${ }^{s} \gamma_{k} \notin \mathrm{Cl}_{p_{k}}(\Gamma)$.

Proof. If ${ }^{s} \gamma_{k} \notin \mathrm{GL}(n, \mathbf{Z})$, then there exists a matrix coefficient $\nu$ of ${ }^{s} \gamma_{k}$ such that $\nu \notin \mathbf{Z}$. Taking $p_{k}$ to be a prime occurring in the denominator of $\nu$, it follows that $\nu \notin \mathbf{Z}_{p_{k}}$. As any limit of elements in $\Gamma$ is in $\operatorname{GL}\left(n, \mathbf{Z}_{p}\right),{ }^{s} \gamma_{k} \notin \mathrm{Cl}_{p_{k}}(\Gamma)$. We now consider the alternative when ${ }^{s} \gamma_{k} \in \operatorname{GL}(n, \mathbf{Z})$. According to Theorem 2.1, if ${ }^{s} \gamma_{k} \in$ $\mathrm{GL}(n, \mathbf{Z}) \cap \mathrm{Cl}(\Gamma)$, then ${ }^{s} \gamma_{k} \in \Gamma$. However, $\Gamma$ is torsion free and ${ }^{s} \gamma_{k}$ is finite order, and thus could not possibly reside in $\Gamma$. Therefore, there must exist a prime $p_{k}$ such that ${ }^{s} \gamma_{k} \notin \mathrm{Cl}_{p_{k}}(\Gamma)$, as desired.

3.1.4. Proof of Theorem 1.1. We are now ready to prove Theorem 1.1 .

Proof of Theorem 1.1. Let $[\eta]$ be a $\operatorname{GL}(n, \mathbf{Z})$-conjugacy class for a semisimple element $\eta$ in $\mathrm{GL}(n, \mathbf{Z})$. Using the primes in Lemma 3.5 and setting

$$
N=\prod_{i=1}^{r} p_{i}, \quad \mathrm{Cl}_{N}(\Gamma)=\prod_{i=1}^{r} \mathrm{Cl}_{p_{i}}(\Gamma)
$$

it follows that ${ }^{s} \gamma_{k} \notin \mathrm{Cl}_{N}(\Gamma)$ for all $k=1, \ldots r$. In particular, $\mathrm{Cl}_{N}(\Gamma)$ contains no semisimple elements by Proposition 3.4. By Lemma 3.2, $\mathrm{Cl}_{N}([\eta])$ consists entirely of semisimple elements. These two facts imply that $\mathrm{Cl}_{N}(\Gamma) \cap \mathrm{Cl}_{N}([\eta])=\emptyset$. By Lemma 3.3 there exists a positive integer $K$ such that $r_{N^{K}}(\Gamma) \cap r_{N^{K}}([\eta])=\emptyset$. The proof is completed by taking the finite index subgroup $r_{N^{K}}^{-1}\left(r_{N^{K}}(\Gamma)\right)$ for $\Gamma_{0}$.

Theorem 1.1 is the strongest possible result. In Section 7.1, we give an example, due to Stebe [21], of an infinite cyclic subgroup of $\operatorname{GL}(n, \mathbf{Z})$ with semisimple generator for which Theorem 1.1 is false. In particular, the virtual unipotency assumption cannot be dropped. 
3.2. Torsion in profinite groups. For a torsion free, residually finite $G$, there is no reason to expect the profinite closure $\widehat{G}$ of $G$ to be torsion free. Indeed, torsion free, finite index subgroups of $\operatorname{GL}(n, \mathbf{Z})$ with $n>2$ provide linear examples (see [13]). Even for nilpotent groups $G$, it need not be the case that $\widehat{G}$ is torsion free (see [10]). However, for the class of $\Gamma$ considered here, it follows from 10 that $\widehat{\Gamma}$ is torsion free. In addition, it follows from [15] that $\mathrm{Cl}(\Gamma)=\widehat{\Gamma}$. Using this with Lemma 3.2 provides a different proof of Corollary 1.2. Our proof of Theorem 1.1 provides an elementary proof that $\widehat{\Gamma}$ is torsion free for virtually unipotent subgroups of $\operatorname{GL}(n, \mathbf{Z})$. In fact, our proof shows that $\mathrm{Cl}(\Gamma)$ contains no semisimple elements.

\section{Proof of Lemma 2.2}

In this section, we prove Lemma 2.2. We refer the reader to 6, for the material used below on nilpotent Lie groups, Lie algebras, and almost crystallographic groups.

4.1. Preliminaries. For a virtually unipotent subgroup $\Gamma$ of $\operatorname{GL}(n, \mathbf{Z})$, there exists a short exact sequence

$$
1 \longrightarrow \Gamma_{u} \longrightarrow \Gamma \longrightarrow \theta \longrightarrow 1
$$

where $\Gamma_{u}$ is the Fitting subgroup of $\Gamma$ and $\theta$ is a finite group (the holonomy group of $\Gamma)$. The associated holonomy representation $\varphi: \theta \rightarrow \operatorname{Out}\left(\Gamma_{u}\right)$ together with a $2-$ cocycle $f \in H_{\varphi}^{2}\left(\Gamma_{u}, \theta\right)$ determine $\Gamma$. We will prove Lemma 2.2 by induction of the step size of $\Gamma_{u}$. The base case when $\Gamma_{u}$ is abelian is simply the case when $\Gamma$ is a crystallographic group. Before addressing the base case, we simplify our situation.

Set $\mathbf{N}$ to be the Mal'cev completion [6, p. 9] of $\Gamma_{u}$ and $\mathfrak{n}$ to be the Lie algebra of $\mathbf{N}$. By construction, $\Gamma_{u}$ admits an injection into $\mathbf{N}$. The group $\mathbf{N}$ is a connected, simply connected, nilpotent Lie group and so the exponential map (see [6, p. 78]) $\exp : \mathfrak{n} \rightarrow \mathbf{N}$ has a smooth inverse $\log : \mathbf{N} \rightarrow \mathfrak{n}$. By Mal'cev rigidity [6, Theorem 1.2.3], the holonomy representation $\varphi$ has a unique extension $\bar{\varphi}: \theta \rightarrow \operatorname{Out}(\mathbf{N})$ and this extension lifts to a homomorphism into $\operatorname{Aut}(\mathbf{N})([\underline{6}$, Lemma 3.1.2]). This provides us with an injection $\psi: \Gamma \rightarrow \mathbf{N} \rtimes_{\bar{\varphi}} \theta$ where, in an abuse of notation, $\bar{\varphi}$ denotes some lift of $\bar{\varphi}$ to $\operatorname{Aut}(\mathbf{N})$. This allows us to write each element $\gamma \in \Gamma$ as $\left(n_{\gamma}, \theta_{\gamma}\right)$ with $n_{\gamma} \in \mathbf{N}$ and $\theta_{\gamma} \in \theta$. We also have a Jordan decomposition of $\gamma$ given by $\gamma=\left(n_{s}, \theta_{\gamma}\right) \cdot\left(n_{u}, 1\right)$ where $n_{s}, n_{\gamma} \in \mathbf{N}$ and $\theta_{\gamma}\left(n_{u}\right)=n_{u}$. The set of semisimple factors under this decomposition is given by

$$
\operatorname{Semi}_{\mathbf{N}}(\Gamma)=\left\{\left(n_{s}, \theta_{\gamma}\right): \gamma \in \Gamma\right\} \subset \mathbf{N} \rtimes_{\bar{\varphi}} \theta
$$

and we can reduce the finiteness of $\operatorname{Semi}(\Gamma) / \Gamma$ to the finiteness of $\operatorname{Semi}_{\mathbf{N}}(\Gamma) / \Gamma$. That this can be done is seen by the following argument. By Mal'cev rigidity, the inclusion of $\Gamma$ into $\operatorname{GL}(n, \mathbf{Z})$ induces a smooth injection $\rho: \mathbf{N} \rtimes_{\bar{\varphi}} \theta \rightarrow \operatorname{GL}(n, \mathbf{R})$. By the uniqueness of the Jordan decomposition (see 3. I.4]), we have that $\rho\left(\left(n_{s}, \theta_{\gamma}\right)\right)=\gamma_{s}$, $\rho\left(\left(n_{u}, 1\right)\right)=\gamma_{u}$. Consequently, it suffices to show the finiteness of $\operatorname{Semi}_{\mathbf{N}}(\Gamma) / \Gamma$. We are now ready to prove Lemma 2.2 .

4.2. Proof of Lemma 2.2. Our proof will be done by inducting on the step size of $\Gamma_{u}$.

Case (Base). In this case $\Gamma_{u} \cong \mathbf{Z}^{m}$ for some $m$ and hence $\mathbf{N}=\mathbf{R}^{m}$. By the Bieberbach theorems (see [4]), we write elements of $\Gamma$ as $(t, S)$ where $t \in \mathbf{Z}^{m}$ and $S \in \mathrm{GL}(m, \mathbf{Z})$. As there are only finitely many $S$ (these are the elements of $\theta$ ), it 
suffices to prove that there are only finitely many semisimple factors of the form $\left(t_{s}, S\right)$ up to $\Gamma$-conjugation for each individual $S$. The action of $S$ on $\mathbf{Q}^{m}$ decomposes into two subspaces $\mathbf{Q}^{m}=W_{S} \oplus W_{t r i v, S}$ where $W_{t r i v, S}$ is the maximal subspace of $\mathbf{Q}^{m}$ on which $S$ acts trivially. It is a simple matter to see that the Jordan decomposition of an element $(t, S)$ is of the form $\left(t_{s}, S\right)\left(t_{u}, I_{m}\right)$ where $t_{s} \in W_{S}$ and $t_{u} \in W_{\text {triv }, S}$. Conjugating by $\left(t, I_{m}\right)$ produces $\left(t_{s}+\left(S-I_{m}\right) t, S\right)$. As we are only concerned with those vectors in $W_{S}$, we may assume $t \in W_{S}$. The possible vectors $t$ form a finite index $\mathbf{Z}$-submodule of $W_{S}(\mathbf{Z})$ whose image under $S-I_{m}$ is still a finite index $\mathbf{Z}$ submodule of $W_{S}(\mathbf{Z})$ since $S-I_{m}$ is invertible on $W_{S}$. As the set of possible vectors $t_{s}$ is contained in $W_{S}(\mathbf{Z})$, up to $\Gamma_{u}$-conjugacy, the possible vectors are identified with a subset of the quotient $W_{S}(\mathbf{Z}) /\left(S-I_{m}\right)(L)$, where $L$ is the $\mathbf{Z}$-submodule of vectors in $W_{S}(\mathbf{Z})$ which arise as translation vectors for an element of $\Gamma_{u}$. As this quotient is finite, we conclude $\operatorname{Semi}_{\mathbf{N}}(\Gamma) / \Gamma_{u}$ is finite and thus $\operatorname{Semi}_{\mathbf{N}}(\Gamma) / \Gamma$ is finite.

Case (General). For the general case, let $\Gamma_{u}^{k}$ denote the $k$ th term in the lower central series for $\Gamma_{u}$ and assume that $\Gamma_{u}$ has step size $j>1$ (i.e., $\Gamma_{u}^{j}=\{1\}$ ). Associated to each $\Gamma_{u}^{k}$ is it's Mal'cev completion $\mathbf{N}_{k}$ and Lie algebra $\mathfrak{n}_{k}$. The conjugate action of $\Gamma$ on $\mathbf{N} \rtimes_{\bar{\varphi}} \theta$ induces an $\operatorname{Ad}(\Gamma)$-action on $\mathfrak{n} \rtimes_{\bar{\varphi}} \theta$. The semisimple factor set $\operatorname{Semi}_{\mathbf{N}}(\Gamma)$ produces a corresponding set

$$
\operatorname{Semi}_{\mathfrak{n}}(\Gamma)=\left\{\left(\eta_{s}, \theta_{\gamma}\right): \gamma \in \Gamma, \eta_{s}=\log \left(n_{s}\right)\right\} \subset \mathfrak{n} \rtimes_{\bar{\varphi}} \theta .
$$

The finiteness of $\operatorname{Semi}_{\mathbf{N}}(\Gamma) / \Gamma$ is equivalent to the finiteness of $\operatorname{Semi}_{\mathfrak{n}}(\Gamma) / \operatorname{Ad}(\Gamma)$. Consequently, it suffices to show the latter. In addition, it suffices to show the finiteness of $\operatorname{Semi}_{\mathfrak{n}}(\Gamma) / \operatorname{Ad}\left(\Gamma_{u}\right)$ as $\left|\operatorname{Semi}_{\mathfrak{n}}(\Gamma) / \operatorname{Ad}\left(\Gamma_{u}\right)\right|$ is at least as big as $\left|\operatorname{Semi}_{\mathfrak{n}}(\Gamma) / \operatorname{Ad}(\Gamma)\right|$. We now establish the finiteness of the latter set. The Lie algebra $\mathfrak{n}$ of $\mathbf{N}$ is a graded vector space of the form

$$
\mathfrak{n}=\bigoplus_{i=0}^{j-1} \mathfrak{n}_{i} / \mathfrak{n}_{i+1}=\bigoplus_{i=0}^{j-1} \operatorname{Gr}_{i}(\mathfrak{n})
$$

where $\mathfrak{n}_{0}=\mathfrak{n}$ and $\mathfrak{n}_{j}=\{0\}$. In particular, each element $\eta_{s}$ has the form

$$
\eta_{s}=\left(\eta_{0}, \ldots, \eta_{j-1}\right), \quad \eta_{i} \in \mathrm{Gr}_{i}(\mathfrak{n})
$$

Notice that we have a pair of almost crystallographic groups $\Gamma^{\prime}$ and $\Gamma^{\prime \prime}$ given by

$$
1 \longrightarrow \Gamma_{u}^{1} \longrightarrow \Gamma^{\prime} \longrightarrow \theta \longrightarrow 1
$$

and

$$
1 \longrightarrow \Gamma_{u} / \Gamma_{u}^{1} \longrightarrow \Gamma^{\prime \prime} \longrightarrow \theta \longrightarrow 1 \text {. }
$$

This pair of groups inject into the groups $\mathbf{N}_{1} \rtimes_{\bar{\varphi}} \theta$ and $\left(\mathbf{N} / \mathbf{N}_{1}\right) \rtimes_{\bar{\varphi}} \theta$, respectively. For $\Gamma^{\prime}$, we have an induced $\operatorname{Ad}\left(\Gamma_{u}^{1}\right)$-action on $\mathfrak{n}_{1} \rtimes_{\bar{\varphi}} \theta$ where the latter is nothing more than

$$
\mathfrak{n}_{1}=\bigoplus_{i=1}^{j-1} \mathfrak{n}_{i} / \mathfrak{n}_{i+1}=\bigoplus_{i=1}^{j-1} \operatorname{Gr}(\mathfrak{n}) .
$$

Likewise, we have an $\operatorname{Ad}\left(\Gamma_{u} / \Gamma_{u}^{1}\right)$-action on $\left(\mathfrak{n} / \mathfrak{n}_{1}\right) \rtimes_{\bar{\varphi}} \theta$. According to our induction hypothesis, there are only finitely many possibilities for $\eta_{1}, \ldots, \eta_{j-1}$ in $(2)$ up to the $\operatorname{Ad}\left(\Gamma_{u}^{1}\right)$-action. Similarly, by our induction hypothesis, there are only finitely many possibilities for $\eta_{0}$ in 2 up to the $\operatorname{Ad}\left(\Gamma_{u} / \Gamma_{u}^{1}\right)$-action. Thus, there are only finitely many possibilities for $\eta_{0}, \ldots, \eta_{j-1}$ in $(2)$ up to the $\operatorname{Ad}\left(\Gamma_{u}\right)$-action. In particular, up 
to the $\operatorname{Ad}\left(\Gamma_{u}\right)$-action, there are only finitely many possibilities for $\eta_{s}$ in $\left(\eta_{s}, \theta_{\gamma}\right) \in$ $\operatorname{Semi}_{\mathfrak{n}}(\Gamma)$. As the possibilities for $\theta_{\gamma}$ range over the finite group $\theta$, this implies the finiteness of $\operatorname{Semi}_{\mathfrak{n}}(\Gamma) / \Gamma$.

\section{Theorem 1.1 for arithmetic lattices}

The proof of Theorem 1.1 and its Corollary 1.2 work for subgroups $\triangle$ of $\operatorname{GL}(n, \mathbf{Q})$ commensurable with GL $(n, \mathbf{Z})$. Briefly we describe this here. We begin with the following lemma whose validity can be deduced from the proof that $\Gamma$ injects into $\mathbf{N} \rtimes_{\bar{\varphi}} \theta$.

Lemma 5.1. There exists a lattice $\Gamma_{0}<\mathbf{N} \rtimes_{\bar{\varphi}} \theta$ such that $\Gamma_{0}$ contains each ${ }^{s} \gamma_{k}$ and $\Gamma$.

With Lemma 5.1, we can generalize Theorem 1.1. To this end, let $\triangle$ be a subgroup of $\mathrm{GL}(n, \mathbf{Q})$ commensurable with $\mathrm{GL}(n, \mathbf{Z})$ and assume that $\triangle$ contains a torsion free, virtually unipotent subgroup $\Gamma$. Using the same approach as in the proof of Theorem 1.1. note that Proposition 3.4 is validated as before (note that passing to convergent subsequences is done now inside the compact set $\left.\mathrm{Cl}_{p}(\triangle)\right)$. For Lemma 3.5, we must modify our argument. It could be the case that $\triangle$ does not contain the elements ${ }^{s} \gamma_{k}$ coming from Lemma 2.2. However, by Lemma 5.1 and 19, Corollary 10.14], there exists a group $\triangle_{0}$ commensurable with $\triangle$ that contains $\Gamma_{0}$. By Theorem 2.1 (this holds for groups commensurable with $\mathrm{GL}(n, \mathbf{Z})), \mathrm{Cl}(\Gamma) \cap \triangle_{0}=\Gamma$. In particular, for each ${ }^{s} \gamma_{k}$, there must exist a prime $p_{k}$ such that ${ }^{s} \gamma_{k} \notin \mathrm{Cl}_{p_{k}}(\Gamma)$. This shows that Theorem 1.1 can be extended to groups $\triangle$ in $\operatorname{GL}(n, \mathbf{Q})$ commensurable with $\mathrm{GL}(n, \mathbf{Z})$. For a general arithmetic lattice $\Lambda$, there exists an injective homomorphism $\psi: \Lambda \rightarrow \operatorname{GL}(n, \mathbf{Q})$ such that $\psi(\Lambda)$ is contained in a subgroup $\triangle$ in $\operatorname{GL}(n, \mathbf{Q})$ that is commensurable with $\operatorname{GL}(n, \mathbf{Z})$. Using the above argument, for any semisimple element $\eta \in \Lambda$, we can find a finite index subgroup $\triangle_{0}$ of $\triangle$ such that $\Gamma<\triangle_{0}$ and $[\eta]_{\triangle} \cap \triangle_{0}=\emptyset$ where $[\eta]_{\triangle}$ is the $\triangle$-conjugacy class of $\eta$. Certainly $[\eta]_{\Lambda} \subset[\eta]_{\triangle}$ and thus $[\eta]_{\Lambda} \cap \triangle_{0}=\emptyset$. Intersecting $\triangle_{0}$ with $\Lambda$ produces a finite index subgroup $\Lambda_{0}$ of $\Lambda$ such that $\Gamma<\Lambda_{0}$ and $[\eta]_{\Lambda} \cap \Lambda_{0}=\emptyset$. In total, we obtain the following theorem and corollary.

Theorem 5.2. Let $\Lambda$ be an arithmetic lattice, $\Gamma<\Lambda$ a torsion free, virtually unipotent subgroup, and $\eta \in \Lambda$ a semisimple element. Then there exists a finite index subgroup $\Lambda_{0}$ of $\Lambda$ such that $\Gamma<\Lambda_{0}$ and $[\eta]_{\Lambda} \cap \Lambda_{0}=\emptyset$.

Corollary 5.3. Let $\Lambda$ be an arithmetic lattice and $\Gamma<\Lambda$ a torsion free, virtually unipotent subgroup. Then there exists a torsion free, finite index subgroup $\Lambda_{0}$ of $\Lambda$ such that $\Gamma<\Lambda_{0}$.

The arithmetic assumption is only used in the proof of Lemma 3.5. Thus, we have the following corollary.

Corollary 5.4. Let $\Lambda<\mathrm{GL}(n, \mathbf{C})$ be a finitely generated group and $\Gamma<\Lambda$ a torsion free, virtually unipotent subgroup. Given an infinite order semisimple element $\eta \in \Lambda$, there exists a finite index subgroup $\Lambda_{0}<\Lambda$ such that $\Gamma<\Lambda_{0}$ and $[\eta]_{\Lambda} \cap \Lambda_{0}=\emptyset$.

This corollary follows from the fact that any semisimple $\eta$ in $\mathrm{Cl}_{p}(\Gamma)$ is conjugate to a torsion element and thus itself is torsion. Indeed, there is nothing special about 
taking the conjugacy class of an infinite order semisimple element. The following is a consequence of the same logic.

Corollary 5.5. Let $\Lambda<\mathrm{GL}(n, \mathbf{C})$ be a finitely generated group, $\Gamma<\Lambda$ a torsion free, virtually unipotent subgroup, and $C$ be an infinite cyclic subgroup generated by a semisimple element. Then $\mathrm{Cl}(\Gamma) \cap \mathrm{Cl}(C)=\{1\}$.

Wilton-Zalesskii have also obtained this result in the case when $\Lambda$ is a Kleinian group and $\Gamma$ is a parabolic subgroup.

Remark. We mention in passing that one can prove Corollary 5.3 as before using the fact that $\widehat{\Gamma}$ is torsion free, $\operatorname{Cl}(\Gamma)=\widehat{\Gamma}$, and Lemma 3.2 .

\section{Geometric applications of Corollary 5.3}

In this section, we derive the main geometric corollaries of Corollary 5.3 mentioned in the introduction. For brevity, we refer the reader to [12, 15, 16] for some of the details.

\subsection{Flat manifolds.}

6.1.1. Proof of Corollary 1.4 For a fixed flat $n$-manifold $N$, Long and Reid 12 , constructed an arithmetic hyperbolic $(n+1)$-orbifold $M$ such that $N$ is diffeomorphic to a cusp cross section of $M$. In particular, $\pi_{1}(N)$ is a torsion free, virtually unipotent subgroup of $\pi_{1}^{\text {orb }}(M)$. By Corollary 5.3 , there exists a finite index, torsion free subgroup $\Lambda_{0}<\pi_{1}^{\text {orb }}(M)$ such that $\pi_{1}(N)<\Lambda_{0}$. Passing to the cover $M_{0} \rightarrow M$ corresponding to $\Lambda_{0}$, yields an arithmetic hyperbolic $(n+1)$-manifold $M_{0}$ such that $N$ is diffeomorphic to a cusp cross section of $M_{0}$.

6.1.2. Nimershiem's conjecture. Reviewing the proof of Corollary 1.4 . notice that passage from $M$ to $M_{0}$ does not change the flat similarity class on the cusp cross section diffeomorphic to $N$. In particular, we obtain the following orbifold-to-manifold promotion.

Corollary 6.1. The space of flat similarity classes on a flat $n$-manifold that arise in cusp cross sections of arithmetic hyperbolic $(n+1)$-orbifolds is precisely the same as those that arise in arithmetic hyperbolic $(n+1)$-manifolds.

We established [16. Proposition 3.2] the density of those similarity classes that arise in cusp cross sections of arithmetic orbifolds. This with Corollary 6.1 proves Theorem 1.3 .

6.1.3. Classification of arithmetic cusp shapes. One of the main motivations for the geometric results of this article come from Gromov [8] whose work inspired the conjectures of Farrell-Zdravkovska [7] and Nimershiem [18. In the former, it was conjectured that every flat $n$-manifold was diffeomorphic to the cusp cross section of a one cusped hyperbolic $(n+1)$-manifold. However, Long and Reid [11 found examples of flat 3-manifolds that can never be diffeomorphic to a cusp cross section of a one cusped hyperbolic 4-manifold. Corollary 1.4 shows the conjectural picture proposed by Farrell-Zdravkovska is not too far off (in some sense).

Corollary 6.1 and [16, Theorem 3.7] show the set of flat similarity classes appearing as cusp shapes in arithmetic hyperbolic manifolds is the image of the rational points 
of an algebraic set under a projection map. In total, this classifies cusp shapes of arithmetic hyperbolic $(n+1)$-manifolds.

6.2. Infranil manifolds. The generalizations to complex and quaternionic hyperbolic spaces of the above results, namely Theorem 1.5. follow from an identical argument using Corollary 5.3 and [16, Theorem 3.5]. The density of these structures in the case $N$ is a Nil 3-manifold follows from Corollary 5.3 and [16, Corollary 3.6]. Finally, Corollary 1.7 follows from Corollary 5.3 and [15, Theorem 3.12]. Just as there are flat $n$-manifolds that cannot arise as the cusp cross section of a single cusped hyperbolic $(n+1)$-manifold, there exist Nil 3-manifolds that cannot arise as the cusp cross section of a one cusped complex hyperbolic 2 -manifold (see 9]). Corollary 1.7 again shows the failure is not total.

\section{Final remarks}

7.1. Generalizing Theorem $\mathbf{5 . 2}$, For a virtually unipotent, torsion free subgroup $\Gamma$, there is no difference in separating $\Gamma$ from an infinite semisimple class or a torsion class. Even for an infinite cyclic group $\langle A\rangle$ generated by a semisimple element $A$, it could very well be the case that one cannot separate $\langle A\rangle$ from a fixed semisimple conjugacy class $[B]$ with $\langle A\rangle \cap[B]=\emptyset$. Indeed, the failure of conjugacy separability in $\mathrm{SL}(n, \mathbf{Z}), n>2$ provides examples (see [21]). Namely, there exists elements $A, B \in$ $\operatorname{SL}(n, \mathbf{Z})$ for $n>2$ such that $A, B$ are not conjugate in $\operatorname{SL}(n, \mathbf{Z})$ but under any homomorphism to a finite group, the images of $A$ and $B$ are conjugate. Note as $A, B$ are conjugate in $\operatorname{SL}\left(n, \mathbf{Z}_{p}\right)$ for all $p,\langle A\rangle$ is disjoint from the $\operatorname{SL}(n, \mathbf{Z})$-conjugacy class of $B$. To see that every finite index subgroup $\Gamma_{0}<\operatorname{SL}(n, \mathbf{Z})$ that contains $\langle A\rangle$ cannot be disjoint from $[B]$, we quotient $\operatorname{SL}(n, \mathbf{Z})$ by the normal core of $\Gamma_{0}$. By our selection of $A, B$, the image of $\Gamma_{0}$ under this homomorphism intersects the image of $[B]$ since the image of $A$ is in the image of $[B]$. Since $\Gamma_{0}$ is the pullback of the image of $\Gamma_{0}$ in this quotient, we see that $\Gamma_{0} \cap[B]$ is non-empty. This shows that one cannot omit the virtual unipotency from Theorem 5.2. It is possible however to separate $\langle A\rangle$ from a fixed torsion class. Indeed, using Theorem 2.1. Lemma 3.2 , and the fact that $\widehat{\langle A\rangle}$ is torsion free, one can find a torsion free finite index $\operatorname{subgroup~of~} \operatorname{SL}(n, \mathbf{Z})$ that contains $\langle A\rangle$. In fact, when $A$ is semisimple, this does not require an arithmetic assumption either.

7.2. Higher rank cusp cross sections. For cusp cross sections of higher rank locally symmetric spaces, the fundamental group of a cusp cross section is virtually solvable but typically not virtually unipotent. For instance, cusp cross sections of Hilbert modular surfaces are Sol 3-orbifolds (see 17 for more on this). Though Theorem 1.1 might not hold for these groups, Corollary 1.2 extends. Indeed, the profinite completion of such torsion free groups are known to be torsion free by 10 . and the profinite completion is isomorphic to $\mathrm{Cl}(\Gamma)$ by $[15$. This with Lemma 3.2 implies Corollary 1.2 for these groups.

7.3. Totally geodesic, immersed surfaces. In general, it seems difficult to resolve torsion in Question 2 from the introduction even when $M$ is a hyperbolic 3-orbifold and $N$ is a totally geodesic surface. However, there are some special cases when this can be done. Indeed, when $\pi_{1}(M)$ is subgroup separable, since $\widehat{\pi_{1}(N)}$ is torsion free 
and the closure of $\pi_{1}(N)$ in $\widehat{\pi_{1}(M)}$ is isomorphic to $\widehat{\pi_{1}(N)}$, one can extend Corollary 1.2. One class of $M$ that satisfy this condition are noncompact arithmetic hyperbolic 3 -orbifolds (see [1]) which are endowed with many totally geodesic, immersed surfaces (see [14]).

Acknowledgements. I wish to thank Karel Dekimpe, Daniel Groves, Christopher Leininger, Gopal Prasad, Alan Reid, Matthew Stover, and Henry Wilton for stimulating conversations and gratefully acknowledge the California Institute of Technology for their hospitality, as part of this work was done while visiting that institution. In addition, I would like to thank the referee for helpful comments, and for improving the clarity and readability of this article.

\section{References}

[1] I. Agol, D. D. Long, and A. W. Reid, The Bianchi groups are separable on geometrically finite subgroups, Ann. of Math. (2) 153 (2001), 599-621.

[2] A. Borel, Compact Clifford-Klein forms of symmetric spaces, Topology 2 (1963) 111-122.

[3] A. Borel, Linear algebraic groups, Springer-Verlag, 1991.

[4] L. S. Charlap, Bieberbach groups and flat manifolds, Springer-Verlag, 1986.

[5] W. W. Crawley-Boevey, P. H. Kropholler, and P. A. Linnell, Torsion-free soluble groups, completions, and the zero divisor conjecture, J. Pure Appl. Algebra 54 (1988), 181-196.

[6] K. Dekimpe, Almost-Bieberbach groups: affine and polynomial structures, Springer-Verlag, 1996.

[7] F. T. Farrell and S. Zdravkovska, Do almost flat manifolds bound?, Michigan Math. J. 30 (1983), 199-208.

[8] M. Gromov, Almost flat manifolds, J. Differential Geom. 13 (1978), 231-241.

[9] Y. Kamishima, Nonexistence of cusp cross-section of one-cusped complete complex hyperbolic manifolds. II. Int. Math. Forum 2 (2007), 1251-1258.

[10] P. Kropholler and J. Wilson, Torsion in profinite completions, J. Pure Appl. Algebra 88 (1993), 143-154.

[11] D. D. Long and A. W. Reid, On the geometric boundaries of hyperbolic 4-manifolds, Geom. Topol. 4 (2000), 171-178.

[12] D. D. Long and A. W. Reid, All flat manifolds are cusps of hyperbolic orbifolds, Algebr. Geom. Topol. 2 (2002), 285-296.

[13] A. Lubotzky, Torsion in profinite completions of torsion-free groups, Quart. J. Math. Oxford Ser. (2) 44 (1993), 327-332.

[14] C. Maclachlan and A. W. Reid, The arithmetic of hyperbolic 3-manifolds, Springer-Verlag, 2003.

[15] D. B. McReynolds, Peripheral separability and cusps of arithmetic hyperbolic orbifolds, Algebr. Geom. Topol. 4 (2004), 721-755.

[16] D. B. McReynolds, Arithmetic cusp shapes are dense, Geom. Dedicata. 129 (2007), 47-55.

[17] D. B. McReynolds, Cusps of Hilbert modular varieties, Math. Proc. Camb. Philos. Soc. 144 (2008), 749-759.

[18] B. E. Nimershiem, All flat three-manifolds appear as cusps of hyperbolic four-manifolds, Topology Appl. 90 (1998), 109-133.

[19] M. S. Raghunathan, Discrete subgroups of Lie groups, Springer-Verlag, 1972.

[20] P. Scott, Subgroups of surface groups are almost geometric, J. London Math. Soc. (2) 17 (1978), 555-565.

[21] P. F. Stebe, Conjugacy separability of groups of integer matrices, Proc. Amer. Math. Soc. 32 (1972), 1-7.

Mathematics, University of Chicago, 5734 S. University, Chicago IL 60637

E-mail address: dmcreyn@math.uchicago.edu

URL: http://math.uchicago.edu/ dmcreyn 